Horm Res 1991;35(suppl 2):V

\title{
Previous Meetings and Presidents of the ESPE
}

\section{2}

Zurich, Switzerland

1977

Cambridge, UK

A. Prader

C.G.D. Brook

1963

Groningen, The Netherlands

1978

Athens, Greece

H.K.A. Visser

C. Dacou-Voutetakis

1964

Hamburg, FRG

1979

Ulm, FRG

J.R. Bierich

W. Teller

1965

Copenhagen, Denmark

1980

Bergamo, Italy

H.J. Andersen

G. Chiumello

1966

Glasgow, UK

1981

Geneva, Switzerland

W. Hamilton 
P.C. Sizonenko

1967

Haifa, Israel

1982

Helsinki, Finland

Z. Laron

J. Perheentupa

1968

Vienna, Austria

1983

Budapest, Hungary

W. Swoboda

F. Peter

1969

Malmö, Sweden

1984

Heidelberg, FRG

C.G. Bergstrand

D. Schönberg

1970

Lyon, France

1985

Baltimore, USA

R. Francois

R. Rappaport

1971

Zurich, Switzerland 1986

Zurich, Switzerland

A. Prader

R. Illig

1972

Louvain, Belgium

1987

Toulouse, France 
P. Malvaux

P. Rochiccioli

1973

Bergen, Norway

1988

Copenhagen, Denmark

D. Aarskog

N. Skakkebaek

1974

Paris, France

1989

Jerusalem, Israel

P. Royer

Z. Laron

1975

Berlin, FRG

1990

Vienna, Austria

H. Helge

H. Frisch

1976

Rotterdam, The Netherlands

1991

Berlin, FRG

H.K.A. Visser

V. Hesse

Joint Meetings of the ESPE and the LWPES

First Joint Meeting 1981 Geneva, Switzerland P.C. Sizonenko

Second Joint Meeting 1985 Baltimore, USA

S. Raiti, C.J. Migeon

Third Joint Meeting 1989 Jerusalem, Israel Z. Laron 